\title{
Design and Implementation of a Network Based Multi- Axis Motion Control System for Laboratory Studies
}

\author{
Y. GÜVEN and S. ATIŞ
}

\begin{abstract}
In this study, an open source network based three axis motion control system has been designed and implemented to be used within motion control courses at engineering and technical branches for applicational studies. This system has been developed by using open source code and suitable physical structure for engineering studies. This experimental set has been evaluated by 107 students in related courses which are electrical machines and servo systems. Students have conducted various experiments by using single, two and three axes respectively. These students have been subjected to a Learner Satisfaction Survey which focuses on cognitive, affective and psychomotor extend of experimental studies in the laboratory with the designed motion control system. It has been observed from the results of this survey that learnercentered approach with the experimental set contribute in learning process positively.
\end{abstract}

Index Terms - Brushless motor, servo system, fieldbus, CAN, Multi-Axis Motion.

\section{INTRODUCTION}

In the field of industrial automation, multi-axis motion control systems (MAMCS) which have two or more synchronized axis are being used in robotics, $\mathrm{CNC}$, printing machines and industrial plants for cutting and packing processes. Compatibility and integration of these systems are essential for production processes which need fast response and flexibility. In order to achieve best coordination, MAMCS's components should have an appropriate communication environment.

Fieldbus technologies are being used for different industrial applications for communication $[1,2]$. The fieldbus is a component that transforms the motion control system into network-based structure. When works on communication and control techniques for motion control system are analysed [38], they indicate that some field bus technologies are made for these particular systems. It also shows that these communication technologies are being consistently developed parallel to other technological developments.

YILMAZ GÜVEN, is with Department of Electronic and Automation University of Kurklareli, Kurklareli, Turkey,(e-mail: ylmzguven@hotmail.com).

(iD https://orcid.org/0000-0002-8205-2490

SELÇUK ATISŞ, is with Department of Electrical and Energy, University of Marmara, İstanbul, Turkey, (e-mail: satis@ marmara.edu.tr).

ID https://orcid.org/0000-0002-2912-9487
Besides communication, many other subjects such as power electronics, kinematics, control theory, artificial intelligence, electrical motors and computer programming are also related with the motion control systems. This relationship with many other fields shows that teaching of motion control systems in engineering and other technical branch is really crucial. Especially in electrics, mechatronics and automation engineering, motion control has become one of the main courses.

Previous studies show how important is teaching of motion control. For example, in long distance education, virtual laboratories are really important. Some works on that can be listed as virtual laboratory structure and its development to show delays on a distributed network based motion control system [9], application to the fuzzy control of a DC Motor [10], motor control demonstration laboratory [11], design and installation of a motion control laboratory that was accessible from web for distance education [12], design of a laboratory which users connected from distance and work with actual hardware [13], connecting to a virtual laboratory from multiple location for saving space and time [14], development of a prototype to control low power brushless DC motor from distance location within server-client structure [15], research and study on educational methods of motion control systems courses [16].

It can be seen that the virtual laboratories have been preferred commonly instead of actual working systems. Virtualization is efficient to some point however it is deficient to solve problems that occurs in actual applications. On the other hand, present systems like robotic arms have unique codes that don't allow users for different application. These structures prevent theoretical and practical studies to come together. We believe that building a MAMCS with open source code is going to help getting over these problems.

In this work, a network based three-axial motion control system has been developed by using open source codes. System is shown in Fig. 1 below. 


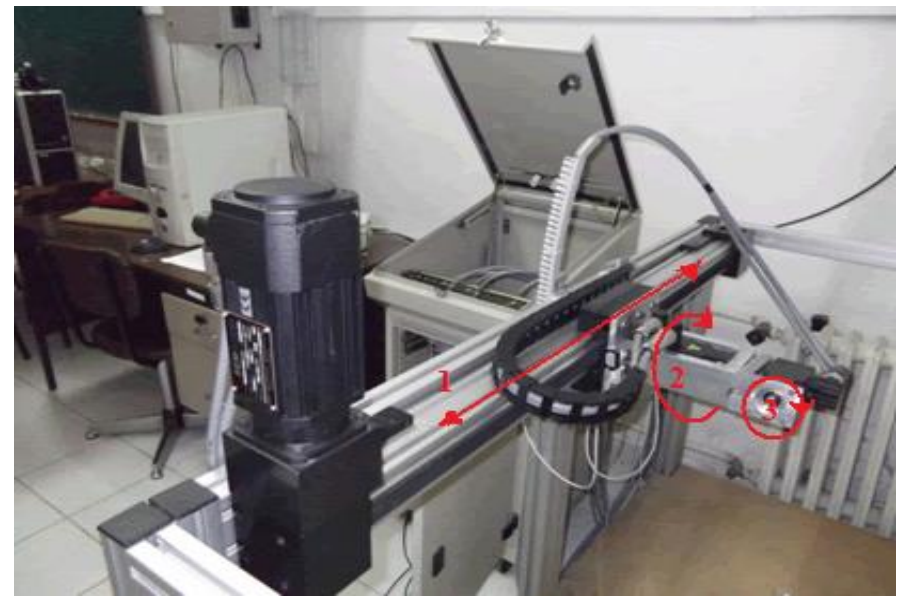

Fig. 1. The network based multi-axial motion control system

The experimental set has brushless servo motors with drivers for each axis and these drivers are being controlled by a network based multi-axis motion controller through CAN-open protocol. Users can access to controller through Ethernet. Controller can be programmed by using a " $\mathrm{C}$ " based programming language and it can be accessed through Telnet as well. Motions can be defined for each axis separately for asynchronous motion and interpolation methods can be used for three axial synchronous linear and circular motions. Parameters of the system can be observed over a web browser and they can be graphically recorded.

Driver can be operated with proportional-integral (PI) control techniques in speed-mode and proportional-integralplus (PIP) control techniques in position-mode. Both techniques can be used together in speed-position-mode and current-control-mode is also active for all these modes. Studies and graphics show that adjusting of parameters of these modes improves stability during applications.

This multi-axial motion control system has been designed to improve success and satisfaction of students in motion control courses as a part of motion control laboratory. A studentcentred approach was used in the design and implementation. The students have been subjected to a Learner Satisfaction Survey which focuses on cognitive, affective and psychomotor extend of experimental studies in the laboratory with the designed motion control system. It has been observed from the results of this survey that learner-centred approach with the experimental set contribute in learning process positively.

\section{DESGN AND IMPLEMANTATION}

\section{A. Design of the system}

The designed MAMCS has three axes with different linear and circular motion ability. The most important requirements of a motion control system are speed and stability. Besides that, production cost and compatibility are other important requirements. Due to these requirements, the brushless direct current motors (BLDC) is selected for their high characteristic specifications. These motors are being driven by high performance digital servo drivers. These drives are also connected to a multi-axis controller for synchronous motions. Communication between drivers and multi-axis controller is established by using CAN-open protocol. Components and connections of network based MAMCS are revealed in Fig. 2.

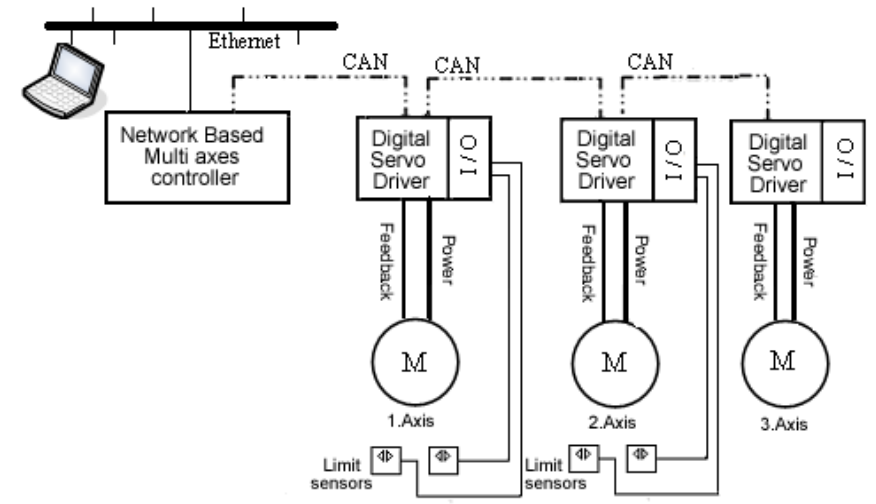

Fig. 2. Components of the MAMCS

The designed mechanical system is defining the main characteristic of the motion. Motion can be continuous or discontinuous as well as it can be linear or circular. Because of that, mechanical system should be designed along with actuator system and sensors. Fig. 3 shows the mechanical system which is designed for different motions both linear and circular. First axis is for converting circular motion of servo motor into linear motion by using high torque geared linear motion module. Second axis is placed with $90^{\circ}$ right angle over this module. Third axis is placed over the second one with same $90^{\circ}$ angle as well to create a three-axial platform.

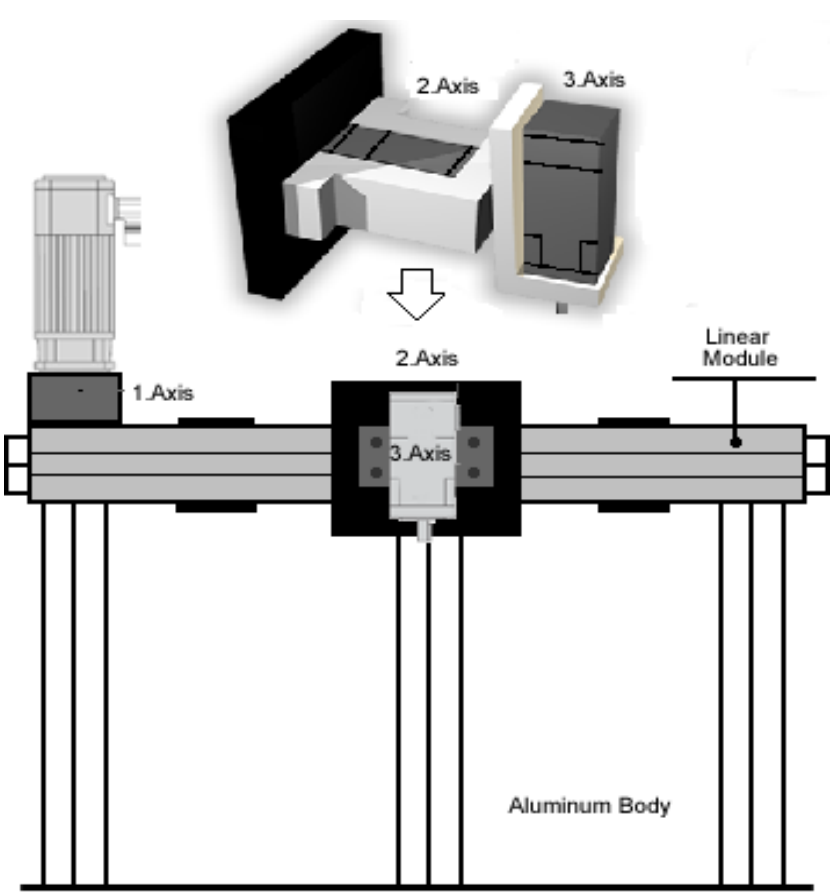

Fig. 3. Schematic of mechanical system. 
Because of weight and vibration issues, aluminium material for main body and flexible strap for the linear motion module is preferred. On the other hand, weight of the module and other mechanism highly effective over power consumption of the system and they also effect response times of the system. Fig. 4 shows the linear motion module and its moving force and moment values are given in Table 1 [17].

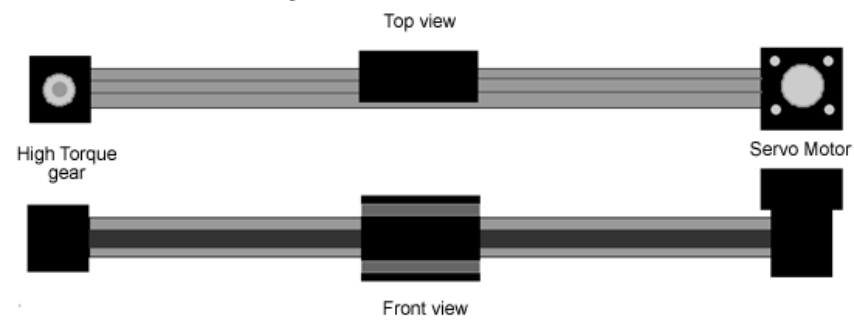

Fig. 4. Top and front view of linear motion module.

Table 1. Dynamic motion values of the linear axis.

\begin{tabular}{lll}
\hline & Unit & Value \\
\hline Fx (Force) & $\mathrm{N}$ & 650 \\
Fy (Force) & $\mathrm{N}$ & 400 \\
Fz (Force) & $\mathrm{N}$ & 1000 \\
$\mathrm{Mx}$ (Moment) & $\mathrm{Nm}$ & 45 \\
$\mathrm{My}$ (Moment) & $\mathrm{Nm}$ & 65 \\
$\mathrm{Mz}$ (Moment) & $\mathrm{Nm}$ & 90 \\
$\mathrm{v} \quad$ (Speed) & $\mathrm{m} / \mathrm{s}$ & 3 \\
\hline
\end{tabular}

As it is shown in Fig. 1 motion platform was placed over aluminium cage and mechanical limits of the system was determined by optical sensors. Placement of optical sensors is shown in Fig. 5.

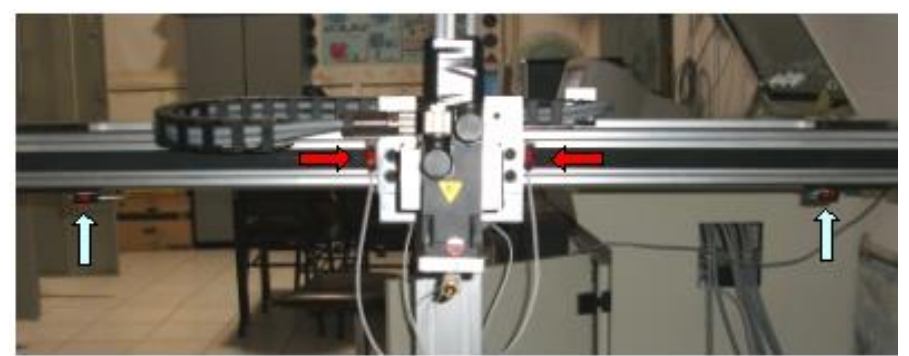

Fig. 5. Optical Sensor placement.

\section{B. Brushless servo motors}

BLDC motors are being widely used in motion control systems because of their high-power capacity within small sizes. Their brushless designs improve their stability and service life. They also have low inertia moment and noise level so they are perfect for robotics and motion control systems [18]. Only disadvantage of these motors is that they need high resolution feedback devices and complicated driver circuits for electronic commutation.

Mathematical model for the system has been created by using mathematical model for brushless servo motor. In this model, some values are accepted as constant value such as space between rotor and stator, magnetic interference, sinusoidal electromagnetic force, friction constant and inertia moment. By this mean, mathematical equation for brushless servo motor is written as shown at Equation (1) and block diagram of the motor is also shown in Fig. 6.

$\left[\begin{array}{c}\frac{d i_{d}}{d t} \\ \frac{d \omega_{m}}{d t}\end{array}\right]=\left[\begin{array}{cc}\frac{-R_{a}}{L_{a}} & -K_{e} \\ \frac{-B}{J} & \frac{1.5 K_{t}}{J}\end{array}\right]\left[\begin{array}{c}i_{d} \\ \omega_{m}\end{array}\right]+\left[\begin{array}{c}\frac{1}{L_{a}} \\ 0\end{array}\right] \cdot V_{d}+\left[\begin{array}{c}0 \\ \frac{-1}{J}\end{array}\right] \cdot T_{1}$

$\begin{array}{clll}i_{d} & \text { : d-axis current } & K_{e} & \text { : Voltage Constant } \\ V_{d} & \text { : d-axis voltage } & K_{t} & \text { : Moment Constant } \\ \omega_{m} & \text { : Angular speed } & B & \text { : Friction Constant } \\ R_{a} & \text { : Coil resistance } & J & \text { : Motor inertia } \\ L_{a} & \text { : Coil Inductance } & T_{l} & \text { : Load moment }\end{array}$

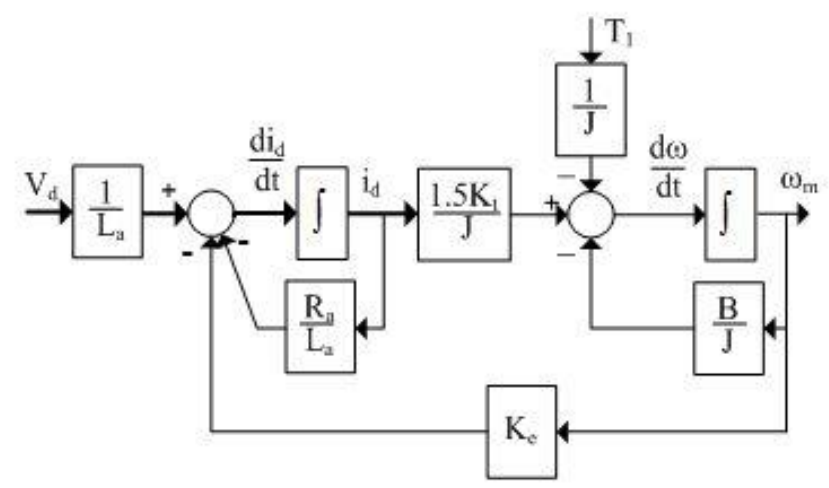

Fig. 6. The block diagram of brushless servo motor

\section{Servo driver}

Brushless motors need a driver circuits to operate. These drivers control electronic commutation, current, speed and position of the motor. Beside that they ensure communication and protection duties [19]. We have preferred to use Digital Signal Processor based servo drivers with high resolution encoder feedback. These drivers have fieldbus communication interface onboard and they support CAN open protocol.

\section{Multi-Axis motion supervisor}

Servo drivers can be connected to a controller for defining synchronous movement with interpolation technique over an open architecture network. We have used a multi-axis motion controller with CAN open protocol support so all device can communicate over an Ethernet network.

\section{E. Communication}

CAN open communication protocol is preferred for this system because of its open architecture. This way all servo drivers can be connected to a single network. CAN open is an open source fieldbus technology that is developed for industrial devices. Communication between multi-axis motion controller and servo drivers has been established by using CAN open protocol. Devices have been connected to each other with line topology and each device has a different node address. 


\section{F. System tunings}

Before defining movement of the axis, initial parameters of the drivers such as proportional $(\mathrm{Kp})$ and Integral gain $(\mathrm{Ki})$ can be assigned by some experiments. For this purpose, the motors have been operated in current, speed and position control mode, respectively. $\mathrm{Kp}, \mathrm{Ki}$ and other system parameters were tuned in this process. System's responses to input commands have been observed graphically. Motor's response of third axis is shown in Fig. 7.

System parameters have been set to get best response, apex point, rising and settlement time. In this point, the most important thing is that system can be set according to applicational requirements such as precision, speed and stability. While determining these parameters, effect of each axis should be also considered in multi-axis motion control systems.

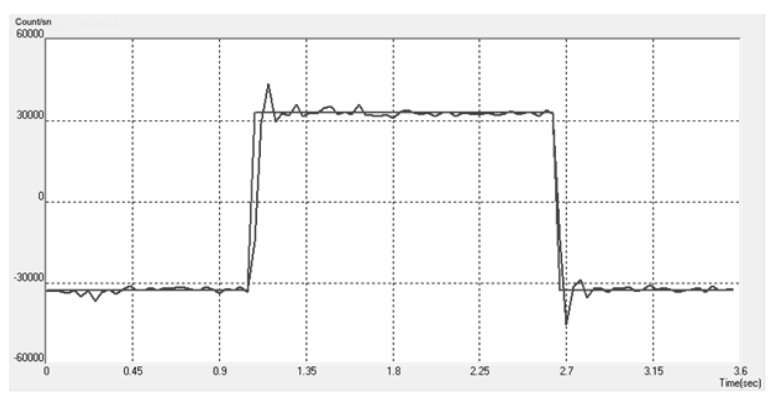

(a)

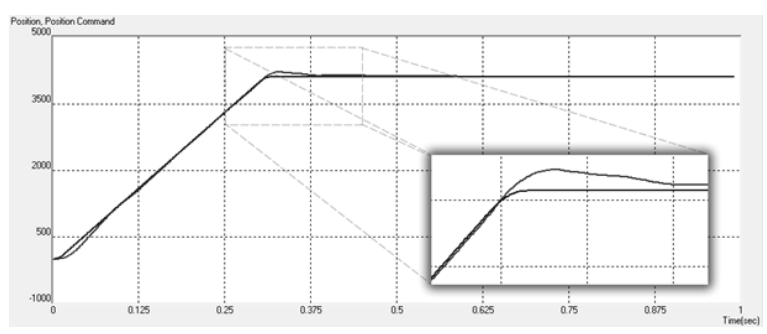

(b)

Fig. 7. Response of the system after tunings

a) speed b) position

\section{EXPERIMENTS}

The experimental set have been evaluated by 107 students in laboratory of motion control systems. Characteristic of these participants can be seen on Table 2 below. As can be seen on the table 2, two different courses which both have application related to servo motors and motion control were subjected to this study. The method was "Experiment in six steps" that is a learner-centred approach as following:

1. In first week, students are informed about the teaching strategies according to learning outputs of the course in the laboratory.

- Experiment groups consist of 3 students

- Establishment of a discussion environment between students and teaching staff during the experiment.

- Preparation and submission of the experiment report as a group.

- Discussion of the experiment results as a group.

2. The students are informed about experiment, laboratory report paper and research topics related to the experiment on the web page.

3. A quiz exam that consist of question about research issues and job security is carried out.

\section{Application and experiment.}

5. After one week from experiment date, the report is delivered to the lecturer. The report is discussed between groups and teaching staffs. Discussion consists of question and answer covering the whole experiment. Preparation of the report as a group is essential.

6. The total score of a student is sum of four different evaluations (quiz exam, performance during the experiment, group score and presentation score of the experiment report) All the stated steps above have been carried out for each experiment in the laboratory. A survey was also applied to measure the effects of the "Experiment in Six Step" approach over students.

Table 2. Participant characteristics.

\begin{tabular}{cccccc}
\hline Course & Participant & Sex & Age & Program & Department \\
\hline $\begin{array}{c}\text { Control of Electrical } \\
\text { Machinery }\end{array}$ & 97 & $\begin{array}{c}10 \text { Female } \\
87 \text { Male }\end{array}$ & $19-22$ & $\begin{array}{c}\text { Associate degree } \\
\text { Bachelor's degree }\end{array}$ & Electrical and Energy \\
\hline Brushless Servo Motors & 10 & 10 Male & $23-25$ & Master's degree & Electrical Eng. \\
\hline
\end{tabular}




\section{RESULTS AND DISCUSSIONS}

PI parameters of the system can be seen at Table 3. These values have been determined while tuning process. They may be changed according to application needs. These parameters are stored in controller's gain table block. Proportional and integral gains are divided by DC voltage value because of PWM duty cycle. This means DC voltage value is used for current controller's gain as well [20]. That is why gain values are high at Table 3.

The students have performed single, two and three axes motion control experiments in the laboratory. Since single axis motion control is the first step of multi-axis motion control, experiments with single axis were excluded in the article.

Table 3. PI parameters for three axes

\begin{tabular}{cccc}
\hline Axis & Name & $\begin{array}{c}\text { Proportional } \\
\text { gain }\left(\mathbf{K}_{\mathbf{p}}\right)\end{array}$ & $\begin{array}{c}\text { Integral gain } \\
\left(\mathbf{K}_{\mathbf{i}}\right)\end{array}$ \\
\hline 1 & a1 & 12.618 & 14363 \\
2 & a2 & 6.581 & 11657 \\
3 & a3 & 6.782 & 12257 \\
\hline
\end{tabular}

The next experimental application with MAMCS was twodimensional interpolated circular movement. Before the experiment some parameters such as synchronization time, maximum acceleration and deceleration values, maximum and minimum speed, smoothness and operation mode should be set. After these settings, a circular movement was defined by its radius, starting angle and trajectory. Fig. 8 shows that a1 as yaxis and $\mathrm{a} 3$ as $\mathrm{x}$-axis for this interpolated circular movement. Position changes and position errors of these two axes are shown in Fig. 9.

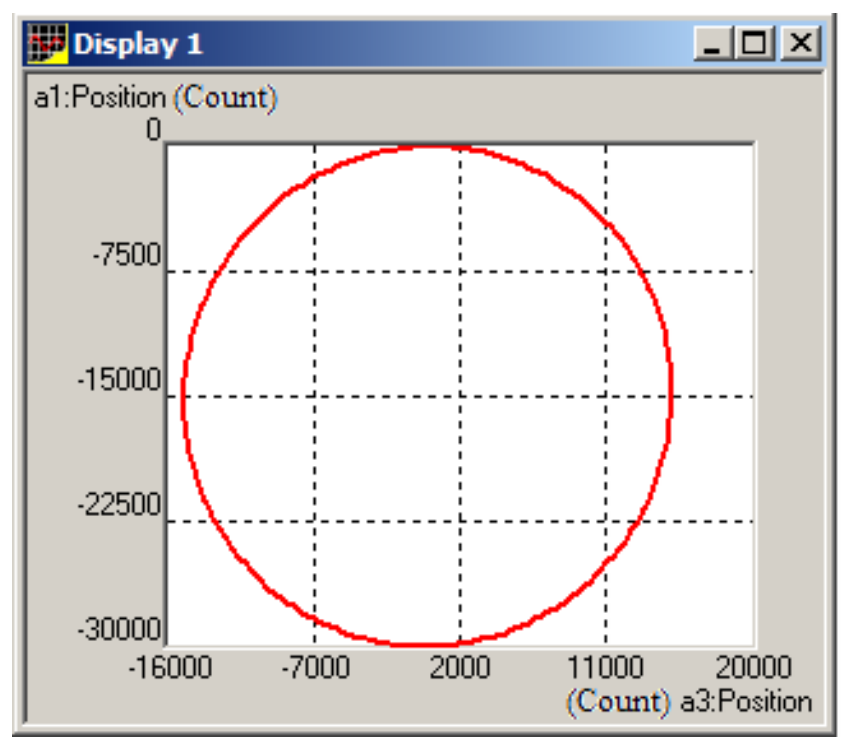

Fig. 8. Circle trajectory.

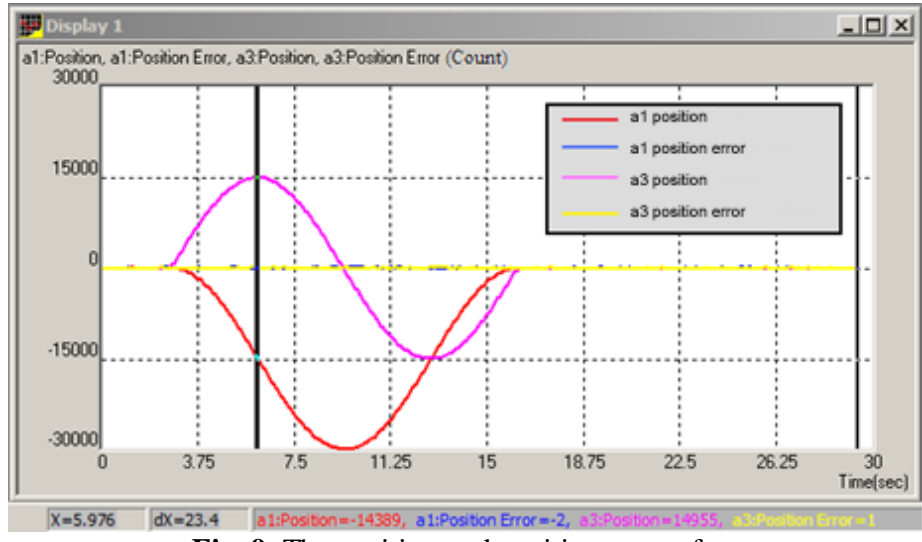

Fig. 9. The position and position error of the $\mathrm{a} 1$ and $\mathrm{a} 3$ axis for circle trajectory.

Another experiment with MAMCS was creating a threedimensional vector with linear interpolation. Same parameters such as synchronization time, maximum acceleration and deceleration, maximum and minimum speed, smoothness and operation mode have set for this movement as well. Two different trajectories have been defined for this linear movement as it is shown at Table 4.

Table 4. The trajectory plane of three-dimensional linear interpolation.

\begin{tabular}{ccccc}
\hline $\begin{array}{l}\text { Axis } \\
\text { No }\end{array}$ & $\begin{array}{l}\text { Axis } \\
\text { Name }\end{array}$ & $\begin{array}{c}\text { Starting } \\
\text { Trajectory } \\
\text { (Count) }\end{array}$ & $\begin{array}{c}\text { First } \\
\text { Target } \\
\text { Trajectory } \\
\text { (Count) }\end{array}$ & $\begin{array}{c}\text { Second } \\
\text { Target } \\
\text { Trajectory } \\
\text { (Count) }\end{array}$ \\
\hline 1 & a1 & 0 & 15000 & 0 \\
2 & a2 & 3850 & -3000 & 0 \\
3 & a3 & 0 & 3000 & 0 \\
\hline
\end{tabular}

Different experiments have been conducted by using different axes. Fig. 10 shows that a1 as $\mathrm{x}$-axis and a2 as $\mathrm{x}$-axis, Fig. 12 shows that a1 as $y$-axis and a2 as $x$-axis and Fig. 14 shows that a1 as $\mathrm{y}$-axis and $\mathrm{a} 3$ as $\mathrm{x}$-axis for changing linear position. Fig. 11, Fig. 13 and Fig. 15 show position and position errors of a1, a 2 and a3. These figures show that position errors for all three axes are very small. 


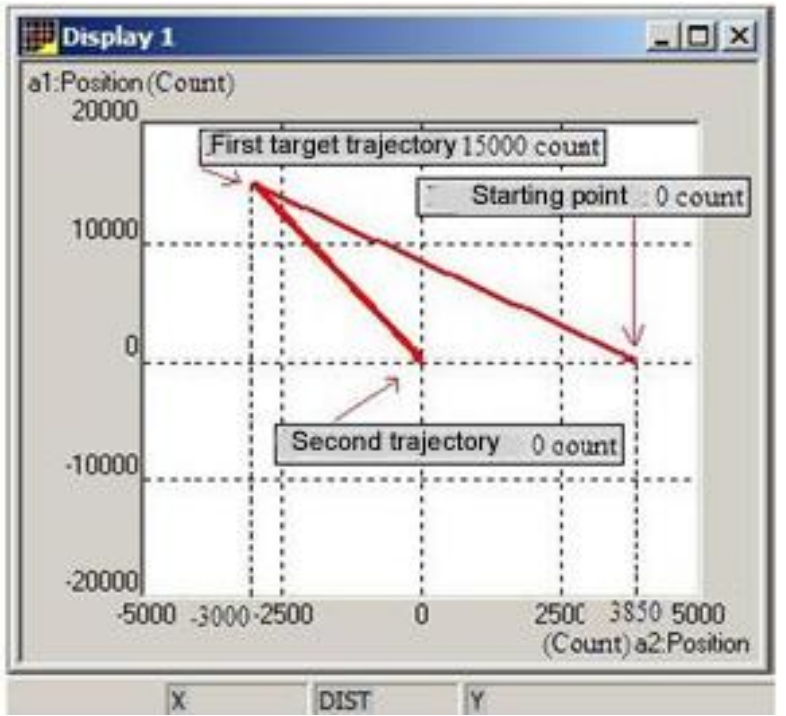

Fig. 10. The line trajectory of the a1-axis.

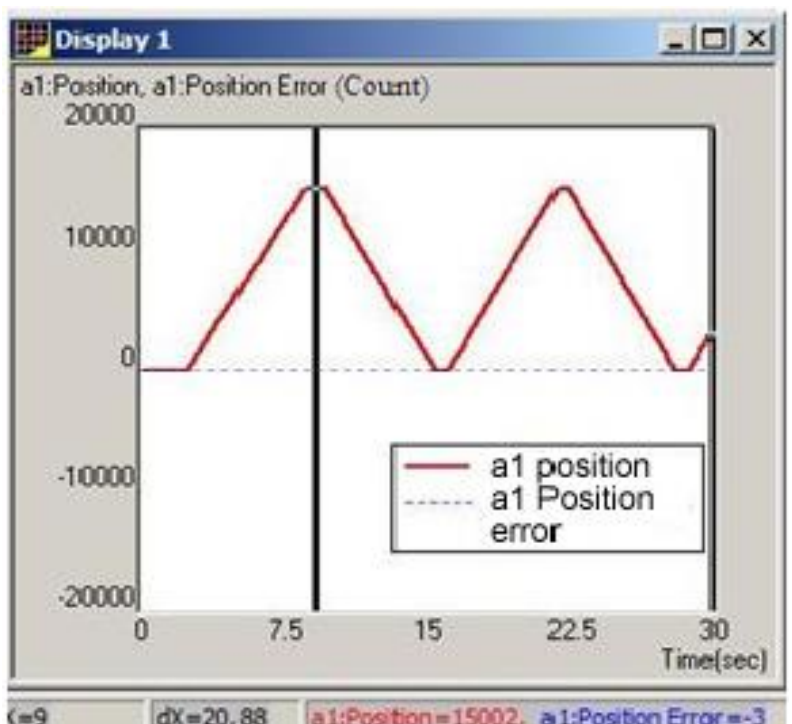

Fig. 11. The position error and position of the a1-axis.

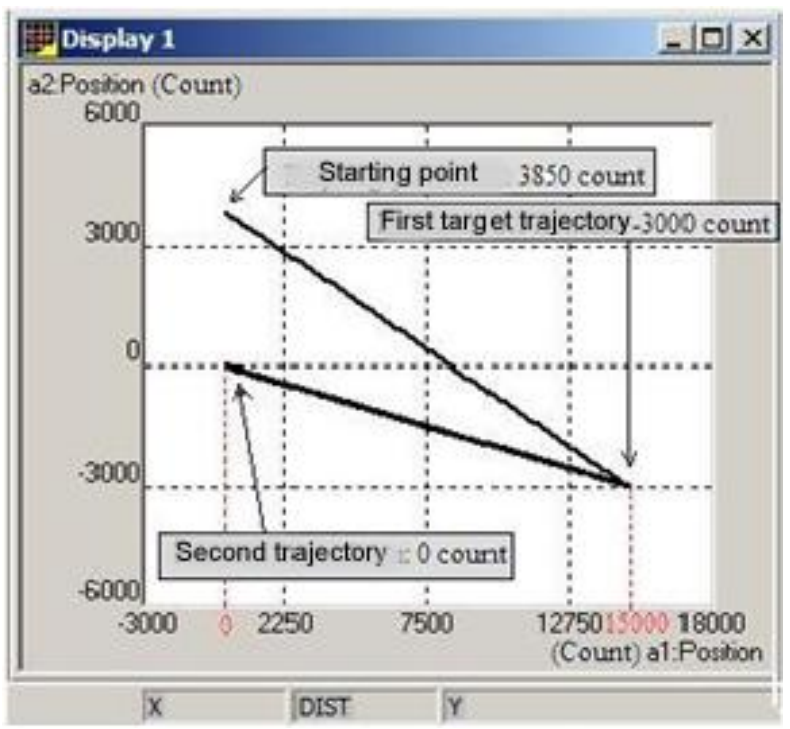

Fig. 12. The line trajectory of the a2-axis.

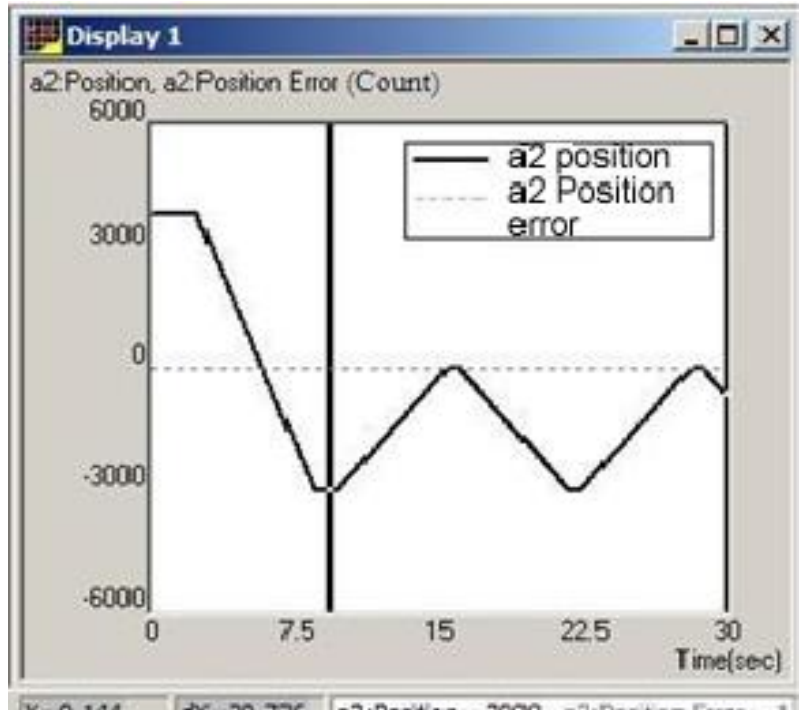

$X=9.144 \quad d X=20.736$ a2:Position=-2999, a2:Position Error $=-1$ Fig. 13. The position error and position of the a2-axis.

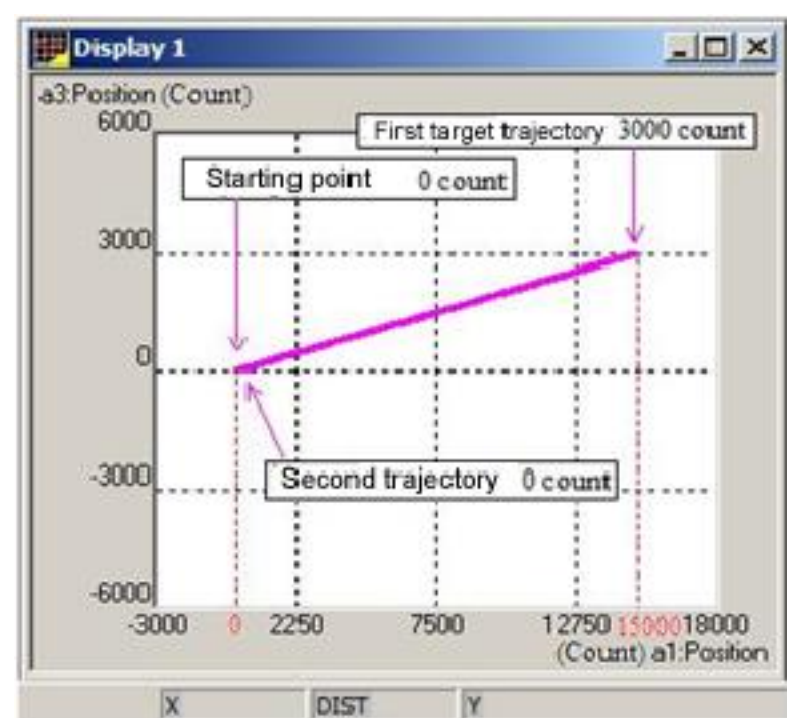

Fig. 14. The line trajectory of the a3-axis.

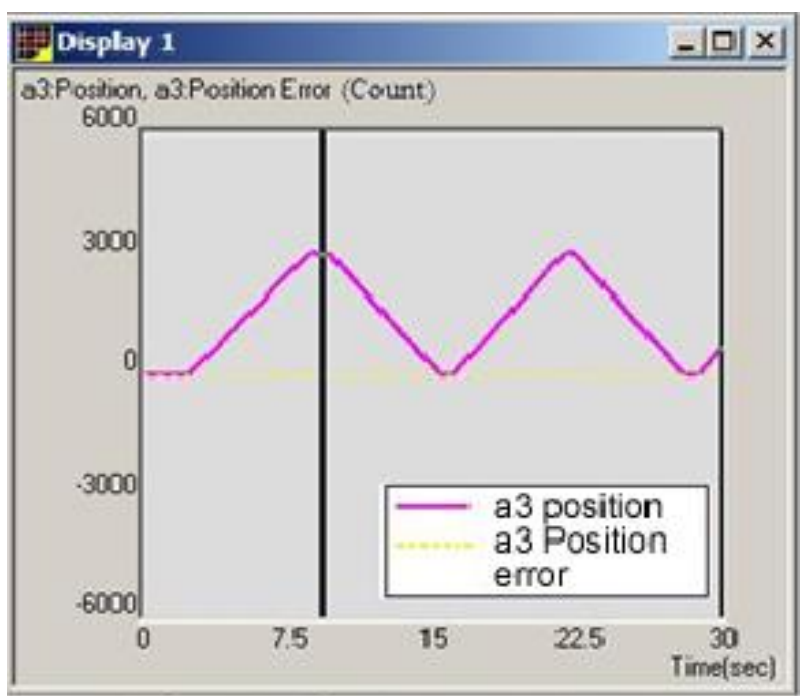

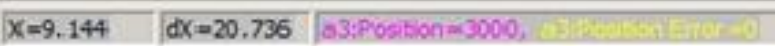

Fig. 15. The position error and position of the a3-axis. 
The last experiment with MAMCS was defined movement trajectory for each axis separately. Application defined in realtime and movement of each axis synchronized. In trajectory planning, parameters like operation modes, speed, acceleration and deceleration, delay time and target trajectory points have been set. Result of this application is revealed in Fig. 16. It shows position commands and errors of all three axis. Graphical data indicates that system settings of MAMCS can be set up within experimental works. This way system works more efficiently and steady compatible with the mechanical system. Accuracy of the systems can be improved by performing different experiments.
The learner-centred approach and the effect of the experimental set on learning process have been measured by questionnaire. Before the survey,

- Single, biaxial and triaxial motion experiments have been conducted. (Excuse week was announced for those who cannot participate on time)

- A technical trip was organized to the production band consisting of robotic arms for Mechatronic engineering students. (Trip were carried out in 5 groups)

A total of 21 question were asked to the students. Students have answered all question on the survey and the result of this survey can be seen through table 5 to 8 .

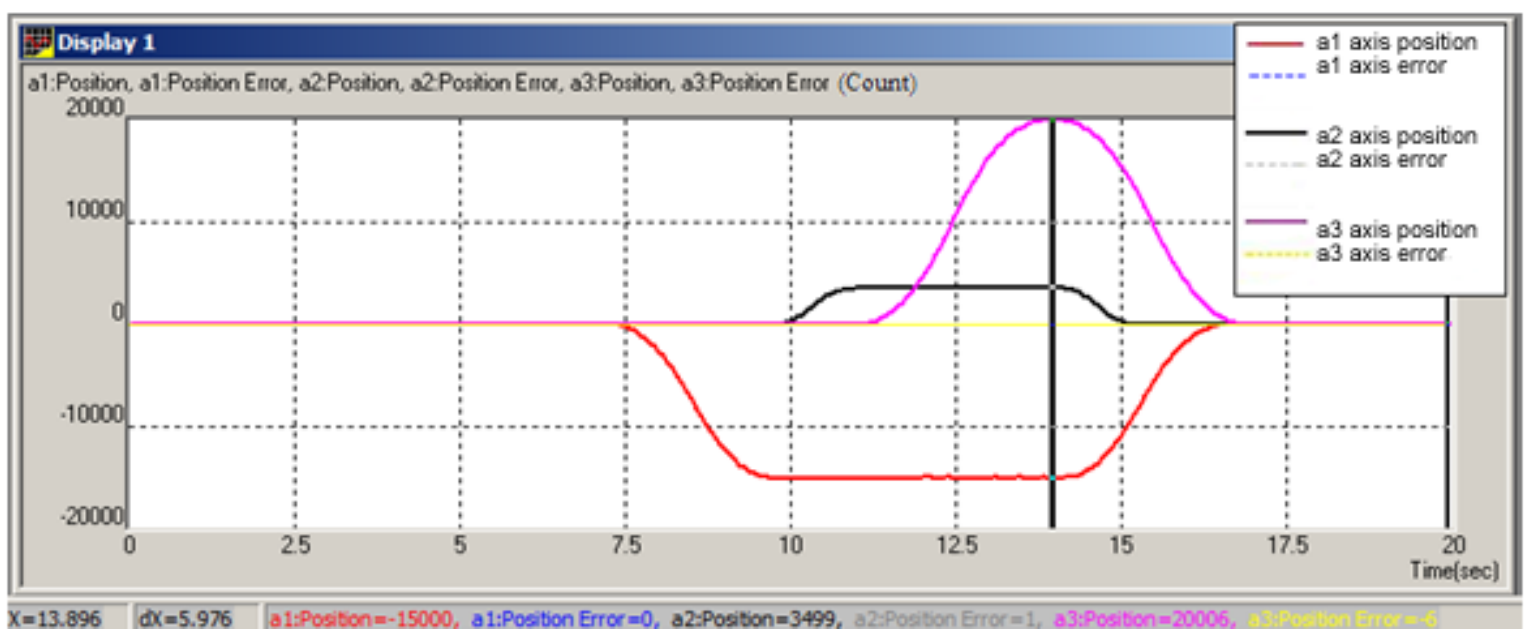

Fig. 16. The position and position error of the a1-a2-a3 ax is for the line trajectory.

Table 5. Results of the learner satisfaction questionnaire 1.

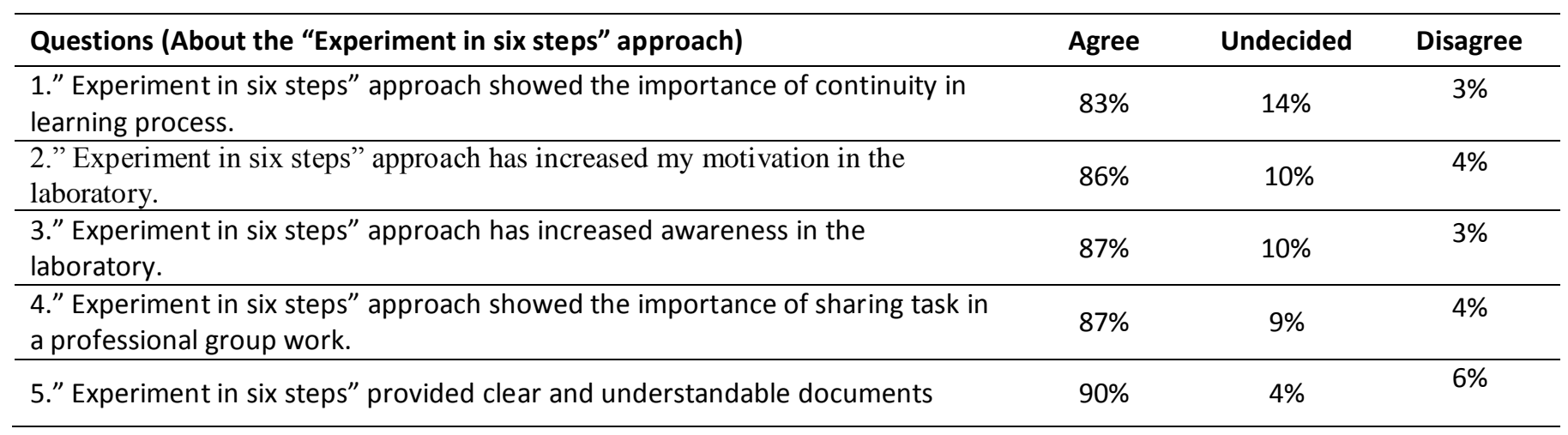

Table 6. Results of the learner satisfaction questionnaire 2 .

\section{Questions (During experiments)}

6. The physical structure of the system is open for user and establishment of the system as a group work is the basis for engineering applications.

\begin{tabular}{lc}
$\begin{array}{l}\text { 7. The system has open source code and programming the system in a group } \\
\text { work were basis for engineering applications. }\end{array}$ & 93 \\
\hline $\begin{array}{l}\text { 8. The relationship between theory and application is clear because of open } \\
\text { source codes and physical structure of the system. }\end{array}$ & 96 \\
\hline
\end{tabular}


Table 7. Results of the learner satisfaction questionnaire 3.

\begin{tabular}{|c|c|c|c|}
\hline Question (after experiments) & 1 axis & 2 axes & 3 axes \\
\hline $\begin{array}{l}\text { 9. I prefer ............... motion control experiment to understand the brushless } \\
\text { servo motor system. }\end{array}$ & $84 \%$ & $4 \%$ & $12 \%$ \\
\hline $\begin{array}{l}\text { 10. I prefer .............. motion control experiment to explain brushless servo } \\
\text { motors. }\end{array}$ & $85 \%$ & $4 \%$ & $11 \%$ \\
\hline $\begin{array}{l}11 . \ldots \ldots \ldots \ldots \ldots \ldots \text { motion control experiment affected my learning performance } \\
\text { more positively. }\end{array}$ & $12 \%$ & $8 \%$ & $80 \%$ \\
\hline $\begin{array}{l}\text { 12. ............ motion control experiment increased my motivation to lesson and } \\
\text { the laboratory. }\end{array}$ & $11 \%$ & $8 \%$ & $81 \%$ \\
\hline $\begin{array}{l}13 . \ldots \ldots \ldots \ldots \text { motion control experiment makes me understand the importance } \\
\text { of task sharing in group work. }\end{array}$ & $6 \%$ & $4 \%$ & $90 \%$ \\
\hline 14. After ............. motion control experiment, I can combine the axis. & $3 \%$ & $5 \%$ & $92 \%$ \\
\hline $\begin{array}{l}\text { 15. After ........... motion control experiment, I can connect drivers and } \\
\text { controller in a multi-axis system. }\end{array}$ & $3 \%$ & $3 \%$ & $94 \%$ \\
\hline $\begin{array}{l}\text { 16. After ............ motion control experiment, I can do linear and circular } \\
\text { trajectory planning. }\end{array}$ & $4 \%$ & $4 \%$ & $92 \%$ \\
\hline
\end{tabular}

Table 8. Results of the learner satisfaction questionnaire 4.

\begin{tabular}{lccc}
\hline Questions (About after the experiments and technical tour) & $\mathbf{1}$ axis & $\mathbf{2}$ axes & $\mathbf{3}$ axes \\
\hline 17. I prefer ........ motion control experiment to explain the robotic systems. & $3 \%$ & $4 \%$ & $93 \%$ \\
\hline 18. I prefer ........ motion control experiment to plan a robotic system. & $9 \%$ & $2 \%$ & $89 \%$ \\
\hline 19. I prefer ........ motion control experiment to apply a robotic system. & $5 \%$ & $3 \%$ & $92 \%$ \\
\hline 20. I prefer ........ motion control experiment to interpret a robotic system. & $5 \%$ & $2 \%$ & $93 \%$ \\
\hline 21. I prefer ........ motion control experiment to analyse a robotic system. & $4 \%$ & $3 \%$ & $93 \%$ \\
\hline
\end{tabular}

Although the "Experiment in six steps" approach led students to a challenging work process, they adopted and found it acceptable for education. That is obvious from the result of the survey (Table 5). This process is up to learner's understanding of importance of continuity, increasing of the motivation and performance of the lecturer.

When the literature is examined, it has been seen that the laboratory of motion control systems was virtual or physically closed to users. This study has shown that the experimental set can easily be used by the students in the laboratory unlike the experiment set in the literature. Table 6 shows the ease of use regarding to experiment set as follows:

- Open source code programming,

- Being physically open to user,

- Studying as a group,

- Students can use their skills and abilities by themselves.

Moreover, the experimental set is completely user-friendly and it reduces the uncertainties to establish strong relationship between theory and practice.
Particularly, it has been understood that observation of robotic system in the technical touring plays a leading role in design and implementation of multi-axis motion control systems as industrial applications. Indication of this guidance can be explained by the fact stated in the previous paragraph and opinions of the students can be seen on table 8 .

The result of the survey has shown that single axis experiments were more useful in understanding the theoretical basis of brushless motors and servo systems. Starting with single axis experiment increases the comprehensibility of the subject. On the other hand, it has been seen that multi-axis experiments were more useful to understand the theoretical basis of multi-axis motion control systems and their industrial application such as CNC machines and robotics. The system can be operated within various industrial modes and students can perform single or multi-axis motion control experiments with interpolation techniques. These abilities play a trigger role in the development of the skills over the students. The preference of the students has been shown in table 7 . 


\section{CONCLUSION}

In this study, an experimental set has been developed to provide a strong link between theoretical knowledge and application in laboratory of motion control system for engineering and other technical branches. The designed network based multi-axis motion control system is suitable for engineering studies and it has physically visible structure and open source code programming.

This user-friendly open source system has been adopted by the students. It has been found that experimental studies with the system are useful for understanding the theoretical basis of multi-axis motion control systems such as CNC machines and robotic arms. The students will not encounter with such a system which is totally open for user both hardware and software. In this point, it is necessary to avoid using completely closed pre-set systems.

The "Experiment in six steps" approach has influenced the motivation of students positively and provided continuity for the experimental process. The awareness and sense of responsibility has increased in the laboratory. The results of the "Experiment in six steps" approach have been questioned by a survey and it has been observed that the experimental set has contributed positively to the cognitive, psychological and psychomotor dimensions of the learning process.

\section{ACKNOWLEDGMENT}

This study has been conducted by using facility of Marmara University, Vocational School of Technical Science and Faculty of Technology.

\section{REFERENCES}

[1] W. Haifang, R. Yu, L. Shengtao, and C. Jinhua, Fieldbus technology and rolling process automation, Computer Design and Applications (ICCDA), 2010 International Conference on, 25-27 June 2010, 2010, pp. 73-76.

[2] Y. Bian, X. Xu, and L. Zhu, Variable frequency hydraulic speed control system based on CANopen,Education Technology and Computer (ICETC), 2010 2nd International Conference on, 22-24 June 2010, 2010, pp. 264-268.

[3] J. Yang, Z. Y. Liu, X. Bi, and Y. Sun, 'The Research and Design of High Precision Contour Machining for Multi-Axis Motion Control', 2015 Chinese Automation Congress (CAC), 2015, pp. 1594-1599.

[4] C. L. Lin, W. H. Chu, and W. Gai, Research on the Synchronization Motion Control Technology for Multi-axis System, 2016 Ieee Information Technology, Networking, Electronic and Automation Control Conference (Itnec), 2016, pp. 672-679.

[5] S. Vitturi, L. Peretti, L. Seno, M. Zigliotto, and C. Zunino, Real-time Ethernet networks for motion control, Computer Standards \& Interfaces, 33(5), 2011, pp. 465-476.

[6] M. R. Jathar, RS-485 based multi axis motor controller,Electronics Computer Technology (ICECT), 2011 3rd International Conference on, 8-10 April 2011, 2011, pp. 104-106.

[7] G. GuoYing, Z. LiMin, X. Zhenhua, and D. Han, Design of a Distributed Multiaxis Motion Control System Using the IEEE-1394 Bus, IEEE Transactions on Industrial Electronics, 57(12), 2010, pp. 4209-4218.

[8] C. Bing, C. You-Ping, X. Jing-Ming, Z. Zu-De, and S. Ji-Ming, Control methodologies in networked motion control systems, Machine Learning and Cybernetics, 2005. Proceedings of 2005 International Conference on, 18-21 Aug. 2005, 2005, pp. 1088-1093.

[9] O. Mirabella, M. Brischetto, and A. Raucea, A network based virtual lab for motion control applications, Advanced Motion Control, 2008. AMC '08. 10th IEEE International Workshop on, 26-28 March 2008, 2008, pp. 342-347.
[10] M. Valles, J. L. Diez, J. L. Navarro, and A. Valera, Remote Access to MATLAB-based Laboratories: Application to the Fuzzy Control of a DC Motor, International Journal of Engineering Education, 26(6), 2010, pp. 1343-1353.

[11] J. Sibigtroth and E. Montanez, Motor control demonstration lab, International Journal of Engineering Education, 21(1), 2005, pp. 84-93.

[12] A. Rojko, D. Hercog, and K. Jezernik, Power Engineering and Motion Control Web Laboratory: Design, Implementation, and Evaluation of Mechatronics Course, IEEE Transactions on Industrial Electronics, 57(10), 2010, pp. 3343-3354.

[13] M. A. Vogelsberger, P. Macheiner, P. Bauer, and T. M. Wolbank, Drives and Motion Control Teaching based on Distance Laboratory and Remote Experiments, Journal of Power Electronics, 10(6), 2010, pp. 579-586.

[14] N. Zhibo, W. Kexin, and D. Chao, Modeling Virtual Laboratory of Motion Control, Computing, Communication, Control, and Management, 2008. CCCM '08. ISECS International Colloquium on, 34 Aug. 2008, 2008, pp. 203-205.

[15] T. Kikuchi, T. Kenjo, and S. Fukuda, Remote laboratory for a brushless DC motor, Education, IEEE Transactions on, 44(2), 2001, p. 12 pp.

[16] G. Lin and C. Fengjun, Research and Discussion on Teaching Methods of Motion Control Systems Course,Circuits, Communications and System (PACCS), 2011 Third Pacific-Asia Conference on, 17-18 July 2011, 2011, pp. 1-4.

[17] Catalog- Linear Modules, Mekatronik Robotik ve Otomasyon Teknolojileri Tic. ve San. Ltd. Sti., 2007.

[18] P. Ki-Hong, K. Tae-Sung, A. Sung-Chan, and H. Dong-Seok, Speed control of high-performance brushless DC motor drives by load torque estimation,Power Electronics Specialist Conference, 2003. PESC '03. 2003 IEEE 34th Annual, 15-19 June 2003, 2003, pp. 1677-1681.

[19] Y. Guven, Three Axial Motion Control with Fieldbus, Master, University of Marmara, 2010.

[20] Elmo Motion Control Ltd, http://www.elmomc.com, Accessed September 2018.

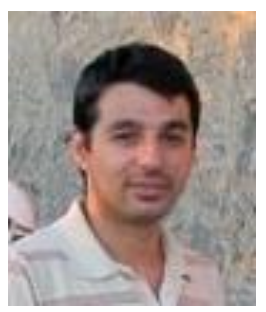

\section{BIOGRAPHIES}

YILMAZ GÜVEN İstanbul, in 1981. He received the B.S. and M.S. degrees in Electrical Education from the University of Marmara, İstanbul, in 2010 and the Ph.D. degree in Electrical Education from Marmara University, İstanbul, in 2018.

From 2012 to 2019, he was a Lecturer with the Vocational School of Technical Science in University of Kirklareli. Since 2019, he has been an Assistant Professor with the Electronic and Automation Department, Kurklareli University. He works on embedded system, data analyses, automation system and electrical machines.

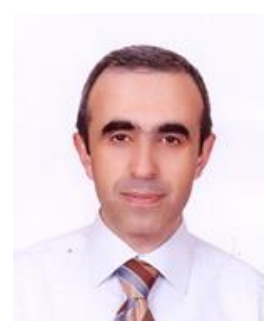

SELÇUK ATIŞ̧ Üsküdar, İstanbul, in 1970. He received the B.S. and M.S. degrees in Electrical Education from the University of Marmara, İstanbul, in 1997 and the Ph.D. degree in Electrical Education from Marmara University, İstanbul, in 2007.

From 1994 to 1998, he was a Research Assistant with the Marmara University. From 1998 to 2009, he was a Lecturer with the Marmara University Since 2009, he has been an Assistant Professor with the Electrical and Energy Department, Marmara University. He works on smart lightning system, expert system and embedded devices. 